\title{
Second Law Analysis of Laminar Flow in a Circular Pipe Immersed in an Isothermal Fluid
}

\author{
Vishal Anand and Krishna Nelanti \\ Infotech Enterprises Limited, Plot No. 2, IT Park, Nanakramguda, Manikonda, Gachibowli, Hyderabad, Andhra Pradesh 500032, India
}

Correspondence should be addressed to Vishal Anand; vish.anand.iit@gmail.com

Received 1 September 2013; Accepted 28 October 2013

Academic Editor: Felix Sharipov

Copyright (C) 2013 V. Anand and K. Nelanti. This is an open access article distributed under the Creative Commons Attribution License, which permits unrestricted use, distribution, and reproduction in any medium, provided the original work is properly cited.

\begin{abstract}
Entropy generation and pumping power to heat transfer ratio (PPR) of a laminar flow, for a circular tube immersed in an isothermal fluid, are studied analytically in this paper. Two different fluids, namely, water and ethylene glycol, are chosen to study the influence of fluid properties on entropy generation and PPR. The expressions for dimensionless entropy generation, Bejan number and PPR are derived in a detailed way and their variations with Reynolds number, external Biot number, and the dimensionless temperature difference are illustrated. The results of the analysis are compared with those for a laminar flow in a circular tube with uniform wall temperature boundary condition. Finally, a criterion is established to determine which type of thermal boundary conditions is more suitable for a particular fluid, with respect to its influence on entropy generation.
\end{abstract}

\section{Introduction}

Heat transfer is a fundamental source of thermodynamic irreversibility in all real engineering devices. When heat is transferred across a finite temperature difference, some capacity to do work is lost. In convection, apart from heat transfer, fluid friction is the other source of loss of available work. Both heat transfer and fluid friction generate entropy. This entropy generation must be minimized to reduce the loss of available work. Entropy generation minimization is no longer an avant-garde philosophy but a mainstream one in the design of thermal systems.

In the past, many researchers have studied the problem of entropy generation minimization in fluid flow with heat transfer. Bejan $[1,2]$ outlined the method for evaluating the entropy generation in fluid flow with heat transfer. $\mathrm{He}$ found out the entropy generated in fluid flow with heat transfer over a flat plate, in a duct, for cylinders in cross flow and in various other geometrical configurations. Sahin [3] studied the entropy generated in a circular duct with uniform wall temperature for two fluids, namely, water and glycerol. The effect of temperature on viscosity was taken into account in that study. He found out that the total energy loss (due to pumping process and entropy generation) can be minimized with respect to the duct length for viscous fluids with temperature dependent viscosity. Şahin $[4,5]$ also considered the effect of duct geometries on the entropy generation, both for uniform wall temperature and for uniform heat flux boundary conditions. The various duct geometries like circular, square, rectangular, equilateral triangle, and sinusoidal were considered. In general, circular geometry was found to be preferable, especially for high Reynolds number. A similar study for hexagonal cross-section was carried out by Jarungthammachote [6] and for a helical coil by Ko and Ting [7]. Dağtekin et al. [8] analyzed the entropy generation in a circular tube with internal fins of different shapes. The study of entropy generation in a pipe was extended to non-Newtonian fluids by Mahmud and Fraser $[9,10]$ and by Hung [11].

As far as the authors are aware, the studies carried out so far have been confined to tubes/ducts with uniform wall temperature or with uniform wall heat flux. The present study is concerned with entropy generation in a circular duct immersed in an isothermal external fluid, which is a more general thermal boundary condition. According to Sparrow and Patankar [12], both the uniform heat flux and uniform wall temperature boundary conditions are the limiting cases of this boundary condition. To study the effect of fluid properties, two different fluids, water and ethylene glycol, have been 


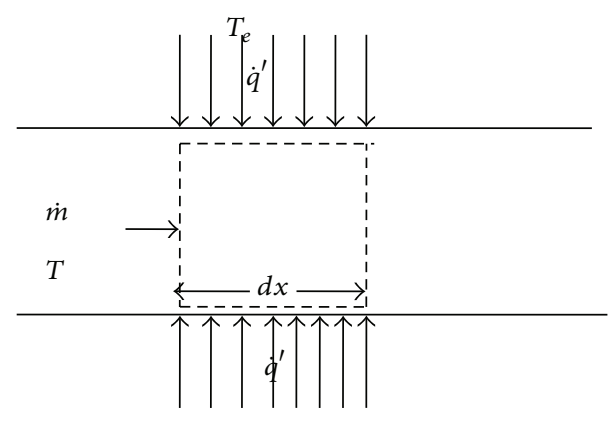

FIgURE 1: An infinitesimal control volume in a circular duct immersed in an isothermal fluid.

considered in this paper. The effects of dimensionless temperature difference and external Biot number on the dimensionless entropy generation, Bejan number, and PPR have been investigated. A comparison has been made with uniform wall temperature boundary condition.

\section{Analysis}

Consider a circular tube immersed in an isothermal fluid (at temperature $T_{e}$ ) as shown in Figure 1. Another fluid flows inside the tube. The flow inside the tube is assumed to be laminar and enters the tube at a uniform temperature $T_{i}$. The energy balance for a control volume (CV) of infinitesimal length $d x$ (neglecting axial conduction, changes in kinetic and potential energies, and viscous dissipation) is given by

$$
\dot{m} C_{p} d T=\dot{q}^{\prime} d x,
$$

where $\dot{q}^{\prime}$, the rate of heat transfer per unit length from the external fluid to the internal fluid, is given by

$$
\dot{q}^{\prime}=\widehat{h} 2 \pi r\left(T_{e}-T\right)
$$

$(\widehat{h}$ in (2) is the overall heat transfer coefficient between the external fluid and the internal fluid).

Solving the two equations above for $T(x)$, we get

$$
T(x)=T_{e}-\left(T_{e}-T_{i}\right) e^{\left(-4 \hat{h} x / \rho U D C_{p}\right)} .
$$

Using nondimensional parameters, we can write this equation as

$$
T(x)=T_{e}-\left(T_{e}-T_{i}\right) e^{(-4 \mathrm{~S} t \lambda)},
$$

where St is the Stanton number given by

$$
\mathrm{St}=\frac{\widehat{h}}{\rho U C_{p}}
$$

and $\lambda$ is the non-dimensional duct length, given by

$$
\lambda=\frac{x}{D} .
$$

The temperature at the outlet of the duct, $T_{\text {out }}$, is given by

$$
T_{\text {out }}=T(L)=T_{e}-\left(T_{e}-T_{i}\right) e^{\left(-4 \mathrm{St} \lambda_{L}\right)},
$$

where

$$
\lambda_{L}=\frac{L}{D}
$$

Writing (7) in terms of mass flow rate, we get

$$
T_{\text {out }}=T(L)=T_{e}-\left(T_{e}-T_{i}\right) e^{\left(-\widehat{\mathrm{N} u k} \pi L / \dot{m} \mathrm{C}_{p}\right)} .
$$

The rate of total heat transferred to the duct is given by

$$
\begin{aligned}
\dot{Q} & =\dot{m} C_{p}\left(T_{\text {out }}-T_{i}\right) \\
& =\dot{m} C_{p}\left(T_{e}-T_{i}-\left(T_{e}-T_{i}\right) e^{-4 \mathrm{St} \lambda_{L}}\right) .
\end{aligned}
$$

The entropy generated in this infinitesimal CV is given by

$$
d \dot{S}_{\text {gen }}=\frac{-\dot{q}^{\prime} d x}{T_{\text {wall }}}+\dot{m} d s
$$

Now,

$$
\begin{aligned}
\dot{q}^{\prime} & =\widehat{h}\left(T_{e}-T\right) \pi D=h\left(T_{\text {wall }}-T\right) \pi D \\
& \Longrightarrow T_{\text {wall }}=\frac{\widehat{h}\left(T_{e}-T\right)}{h}+T .
\end{aligned}
$$

Substituting the values of $\dot{q}^{\prime}$ and $T_{\text {wall }}$ from (12) into (11), we get the following:

$$
d \dot{S}_{\text {gen }}=\frac{-\widehat{h}\left(T_{e}-T\right) \pi D d x}{\widehat{h}\left(T_{e}-T\right) / h+T}+\dot{m} d s .
$$

For an incompressible fluid,

$$
\dot{m} d s=\dot{m} \frac{C_{p} d T}{T}-\dot{m} \frac{d P}{\rho T},
$$

where the pressure drop per unit length is related to the friction factor as

$$
-\frac{d P}{d x}=\frac{f \rho U^{2}}{2 D} .
$$

Thus we get

$$
\dot{m} d s=\frac{\dot{m} C_{p} d T}{T}+\frac{\dot{m} f U^{2}}{2 D T} d x .
$$

Substituting (16) to (13) we get

$$
d \dot{S}_{\text {gen }}=\frac{-\widehat{h}\left(T_{e}-T\right) \pi D d x}{\widehat{h}\left(T_{e}-T\right) / h+T}+\dot{m} C_{p} \frac{d T}{T}+\dot{m} \frac{f U^{2}}{2 D T} d x .
$$

Substituting the value of $T(x)$ from (4) into (17) and integrating (17) from $x=0$ to $x=L$, we get the total entropy generation rate in the duct as

$$
\begin{gathered}
\dot{S}_{\text {gen }}=\dot{m} C_{p}\left[\frac{-1}{\alpha} \ln \left|\frac{1-\tau \alpha e^{-4 \mathrm{St} \lambda_{L}}}{1-\tau \alpha}\right|+\ln \left|\frac{1-\tau e^{-4 \mathrm{St} \lambda_{L}}}{1-\tau}\right|\right. \\
\left.+\frac{f E c \tau}{8 \mathrm{St}} \ln \left|\frac{e^{4 \mathrm{St} \lambda_{L}}-\tau}{1-\tau}\right|\right],
\end{gathered}
$$


where St is the Stanton number (defined in (5)),

$$
\begin{gathered}
\alpha=1-\frac{\widehat{\mathrm{Nu}}}{\mathrm{Nu}}, \\
\tau=\frac{T_{e}-T_{i}}{T_{e}} .
\end{gathered}
$$

Ec is the Eckert number given as

$$
\mathrm{Ec}=\frac{U^{2}}{C_{p}\left(T_{e}-T_{i}\right)} .
$$

In the literature, dimensionless entropy generation has also been used to quantify the entropy generated. It is given by

$$
\begin{aligned}
\psi= & \frac{\dot{S}_{\text {gen }}}{\dot{m} C_{p}}=\frac{-1}{\alpha} \ln \left|\frac{1-\tau \alpha e^{-4 \mathrm{St} \lambda_{L}}}{1-\tau \alpha}\right|+\ln \left|\frac{1-\tau e^{-4 \mathrm{St} \lambda_{L}}}{1-\tau}\right| \\
& +\frac{f \mathrm{Ec} \tau}{8 \mathrm{St}} \ln \left|\frac{e^{4 \mathrm{St} \lambda_{L}}-\tau}{1-\tau}\right|
\end{aligned}
$$

If we take the limit $\alpha \rightarrow 0$, that is, $(\widehat{\mathrm{Nu}} \rightarrow \mathrm{Nu})$, (18) reduces to

$$
\begin{aligned}
\lim _{\alpha \rightarrow 0} \dot{S}_{\text {gen }}=\dot{m} C_{p}\left\{\lim _{\alpha \rightarrow 0}\left(\frac{-1}{\alpha} \ln \left|\frac{1-\tau \alpha e^{-4 \mathrm{St} \lambda_{L}}}{1-\tau \alpha}\right|\right)\right. \\
+\lim _{\alpha \rightarrow 0}\left(\ln \left|\frac{1-\tau e^{-4 \mathrm{St} \lambda_{L}}}{1-\tau}\right|\right) \\
\left.+\lim _{\alpha \rightarrow 0}\left(\frac{f \mathrm{Ec} \tau}{8 \mathrm{St}} \ln \left|\frac{e^{4 \mathrm{St} \lambda_{L}}-\tau}{1-\tau}\right|\right)\right\} .
\end{aligned}
$$

Using the identity $\lim _{a \rightarrow 0}(\ln (1+a) / a)=1$, we get in the simplified form the following:

$$
\begin{aligned}
\lim _{\alpha \rightarrow 0} \dot{S}_{\text {gen }}=\dot{m} C_{p}\{ & -\tau\left(1-e^{-4 \mathrm{St} \lambda_{L}}\right)+\ln \left|\frac{1-\tau e^{-4 \mathrm{St} \lambda_{L}}}{1-\tau}\right| \\
& \left.+\frac{f \tau \mathrm{EC}}{8 \mathrm{St}} \ln \left|\frac{e^{4 \mathrm{St} \lambda_{L}}-\tau}{1-\tau}\right|\right\},
\end{aligned}
$$

which is the same as the expression for entropy generation for fully developed laminar flow in a circular pipe with uniform wall temperature as given in [3]. This corroborates the conclusion of Sparrow and Patankar [12] that, as the external Nusselt number tends to duct side Nusselt number, the isothermal external fluid boundary condition tends to the uniform wall temperature boundary condition.

Equation (22) can also be written as function of Re instead of St $(\mathrm{St}=\widehat{\mathrm{Nu}} / \operatorname{Re} \mathrm{Pr})$ :

$$
\begin{aligned}
\psi= & \frac{-1}{\alpha} \ln \left|\frac{1-\tau \alpha e^{-\pi_{1} / \operatorname{Re}}}{1-\tau \alpha}\right|+\ln \left|\frac{1-\tau e^{-\pi_{1} / R e}}{1-\tau}\right| \\
& +\pi_{2} \operatorname{Re}^{2} \ln \left|\frac{e^{\pi_{1} / \operatorname{Re}}-\tau}{1-\tau}\right|,
\end{aligned}
$$

where

$$
\begin{gathered}
\pi_{1}=\frac{4 \widehat{\mathrm{Nu}} \lambda_{L} k}{\mu C_{p}}, \\
\pi_{2}=\frac{(f \mathrm{Re}) \mu^{3}}{8 \widehat{\mathrm{Nu}} T_{e}(D \rho)^{2} k} .
\end{gathered}
$$

2.1. Bejan Number. The dimensionless entropy generation gives a good idea of the rate of total entropy generation. However, it does not convey which out of the two entropy generation mechanisms, namely, heat transfer and fluid friction, dominates. To resolve this, Paoletti et al. [13], defined a dimensionless number, called Bejan number, as

$$
\mathrm{Be}=\frac{\text { Entropy Generated Due To Heat Transfer }}{\text { Total Entropy Generated }} .
$$

At $\mathrm{Be}=1$, all the entropies are generated due to heat transfer and at $\mathrm{Be}=0$, all the entropies generated are due to fluid friction.

In the current analysis, if we take $\lim _{T_{\infty} \rightarrow T_{i}} \psi$, it gives us the dimensionless entropy generated due to fluid friction only (as $T_{e} \rightarrow T_{i}$, there is no heat transfer, and so entropy generated will be due to fluid friction only):

$$
\mathrm{FF}=\lim _{T_{e} \rightarrow T_{i}} \psi=\pi_{1} \times \pi_{2} \times \mathrm{Re},
$$

where FF is dimensionless entropy generated due to fluid friction only.

So,

$$
\mathrm{Be}=\frac{\psi-\mathrm{FF}}{\psi}=1-\frac{\mathrm{FF}}{\psi} .
$$

2.2. Pumping Power to Heat Transfer Ratio. Another parameter which is a good indicator of the performance of a heat exchanger pipe is the ratio of pumping power to heat transfer (PPR), which can be expressed as

$$
\operatorname{PPR}=\frac{A \Delta P U}{\dot{Q}}
$$

Here the pressure drop $\Delta P$ along the length of the duct can be obtained by integrating the infinitesimal pressure drop $d P$ given by

$$
d P=\frac{-f \rho U^{2}}{2 D} d x
$$

Using (32) to calculate pressure drop and (10) to calculate heat transfer rate and inserting the same into (31), we get

$$
\operatorname{PPR}=\frac{\mu^{2} \lambda_{L}(f \operatorname{Re})(\operatorname{Re} / \tau)}{2 \rho^{2} D^{2} C_{p} T_{e}\left[1-e^{\left(-\pi_{1} / \mathrm{Re}\right)}\right]} .
$$


2.3. Fluid Properties. To study the effect of fluid properties, two different fluids, water and ethylene glycol, are considered.

Water. The thermophysical properties of water are calculated using the following equations, given in [6]:

$$
\begin{aligned}
& \rho_{w}\left(\mathrm{~kg} / \mathrm{m}^{3}\right)=1000\left(1-\frac{(T+288.9414)(T-3.9863)^{2}}{508929.2(T+68.12963)}\right) \text {, } \\
& k_{w}(\mathrm{~W} / \mathrm{mk})=-0.3838+0.005254 \times(T+273) \\
& -0.000006369 \times(T+273)^{2}, \\
& \mu_{w}\left(\mathrm{Ns} / \mathrm{m}^{2}\right)=\left(\operatorname { e x p } \left[-2.10+(-4.45)\left(\frac{273}{(T+273)}\right)\right.\right. \\
& \left.\left.+6.55\left(\frac{273}{(T+273)}\right)^{2}\right]\right)(0.00179)
\end{aligned}
$$

$$
\begin{aligned}
C_{p w}(\mathrm{~J} / \mathrm{kg} \mathrm{K})= & \left(7.25575005 \times 10^{1}-6.62445402\right. \\
& \times 10^{-1}(T+273)+2.56198746 \\
& \times 10^{-3} 3(T+27)^{2} \\
& -4.3659123 \times 10^{-6}(T+273)^{3} \\
& \left.+2.78178981 \times 10^{-9} 3(T+27)^{4}\right) \times \frac{R}{0.018} .
\end{aligned}
$$

In the above equations, $T$ is the temperature of water in degree $C$ and $R$ is the universal gas constant.

Ethylene Glycol. The density of ethylene glycol is taken as constant, since it varies roughly by only $1 \%$ in the temperature range considered. For determining the viscosity of ethylene glycol, the following empirical correlation given by Sherman [14] is used:

$$
\mu(T)=\mu\left(T_{\text {ref }}\right)\left(\frac{T}{T_{\text {ref }}}\right)^{n} \exp \left[B\left(\frac{1}{T}-\frac{1}{T_{\text {ref }}}\right)\right] .
$$

In (38), $T_{\text {ref }}$ is the reference temperature whose value is taken as 273 K. $B$ and $n$ are fluid dependent parameters, whose numerical values for ethylene glycol are found out using regression. To estimate thermal conductivity and specific heat, the fourth degree polynomial equations are used. The coefficients of these polynomial equations are also determined using regression. The data for regression is taken from [15]. The regression is carried out using the commercially available software Microsoft Excel.

The fluid properties are evaluated at $T_{\mathrm{avg}}=\left(T_{\text {out }}+T_{i}\right) / 2$ using an iterative procedure. A value of $T_{\text {out }}$ is guessed. Using this guessed value, the fluid properties are found out at $T_{\text {avg }}$. These fluid properties are then used to find out the new $T_{\text {out }}$ using (7). This process is repeated till the difference in the successive values of $T_{\text {out }}$ is less than $1 \mathrm{~K}$. The $T_{\text {out }}$ for
TABLE 1: Nu and $\widehat{\mathrm{Nu}}$ as a function of Bi taken from [12].

\begin{tabular}{lcc}
\hline $\mathrm{Bi}$ & $\mathrm{Nu}$ & $\widehat{\mathrm{Nu}}$ \\
\hline 0 & 4.364 & 0 \\
0.1 & 4.330 & 0.1909 \\
0.25 & 4.284 & 0.4471 \\
0.5 & 4.221 & 0.8075 \\
1 & 4.122 & 1.345 \\
2 & 3.997 & 1.998 \\
5 & 3.840 & 2.773 \\
10 & 3.758 & 3.163 \\
100 & 3.663 & 3.597 \\
$\infty$ & 3.657 & 3.657 \\
\hline
\end{tabular}

TABLE 2: Values of different constant parameters used in the analysis.

\begin{tabular}{lc}
\hline Parameters & Numerical values \\
\hline$T_{e}$ & $310 \mathrm{~K}$ \\
$D$ & $0.08 \mathrm{~m}$ \\
$L$ & $1.0 \mathrm{~m}$ \\
\hline
\end{tabular}

the last iteration is taken as the final value. The $T_{\text {avg }}$, which is calculated using the final value of $T_{\text {out }}$, is used to estimate the fluid properties.

\section{Results and Discussion}

The $\mathrm{Nu}$ and $\widehat{\mathrm{N}} \mathrm{u}$ are functions of external $\mathrm{Bi}$. Their values are given in tabular form in [12] and are presented in Table 1. The value of friction factor for laminar flow is taken from [16] and is represented as:

$$
f=\frac{64}{\operatorname{Re}} \text {. }
$$

The numerical values of the constant parameters are given in Table 2. For these parameters, the effect of $\mathrm{Bi}$ and $\tau$ on the dimensionless entropy generation $\psi$, the Bejan number $\mathrm{Be}$, and the PPR has been studied. The results are detailed below.

3.1. Effect on $\psi$. Figure 2 shows the variation of $\psi$ with $\operatorname{Re}$ for different values of (a) $\mathrm{Bi}$ and (b) $\tau$ for water. As can be seen from the figure, $\psi$ decreases with Re. To explain this, we consider (4), where we find that if we let Re tend to infinity, then the $T(x)$ tends to $T_{i}($ since $\mathrm{St}=\mathrm{Nu} /(\operatorname{Re} \cdot \operatorname{Pr}))$; that is,

$$
\lim _{\operatorname{Re} \rightarrow \infty} T(x)=T_{i} .
$$

Therefore, at higher Re the temperature gradients inside the fluid decrease and there is less entropy generated due to heat transfer. Even though as Re increases, the entropy generated due to pressure increases, but as we will see later, the contribution of entropy generated due to pressure to total entropy generated is very less ( $\mathrm{Be}$ is close to 1 ), so $\psi$ decreases when Re increases.

It is also seen from Figure 2(a) that as Re increases, the curves for different Bi tend to merge. Similarly, the curves for 


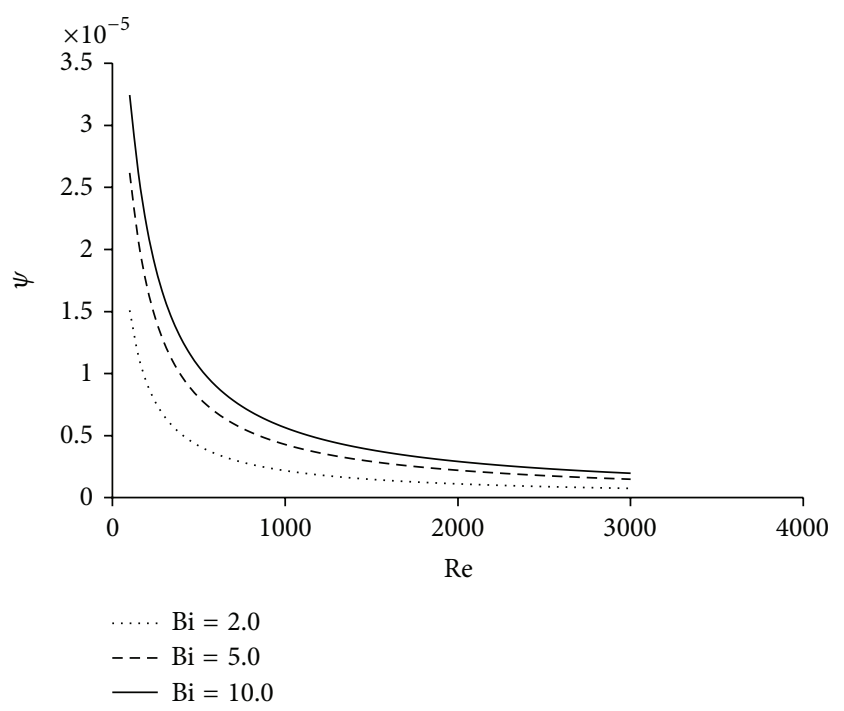

(a)

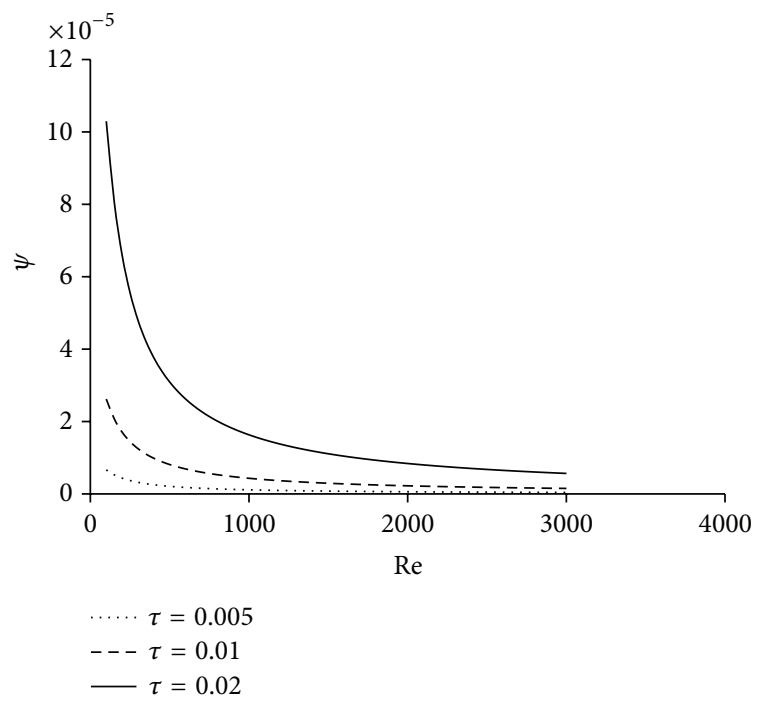

(b)

FIGURE 2: Variation of dimensionless entropy generation $\psi$ of water with Re for different values of (a) Bi and (b) $\tau$.

different $\tau$ also tend to merge at higher Re (see Figure 2(b)). This is because, as Re increases, the contribution of heat transfer to entropy generation decreases and total entropy generated is roughly due to pressure only. Since entropy generation due to pressure is independent of $\mathrm{Bi}$ or $\tau$, the curves for different $\mathrm{Bi}$ (and for different $\tau$ ) tend to merge at higher Re.

We see from Figure 2(a) that $\psi$ increases with increase in $\mathrm{Bi}$. This is because as $\mathrm{Bi}$ increases, $\widehat{\mathrm{N}} \mathrm{u}$ increases (see Table 1 ), which means that heat transfer rate to the fluid increases. This leads to increase in temperature gradients inside the fluid. This increase in temperature gradient inside the fluid leads to increase in entropy generation.

Figure 2(b) shows that, as the dimensionless temperature difference $\tau$ increases, $\psi$ increases. This is because an increase

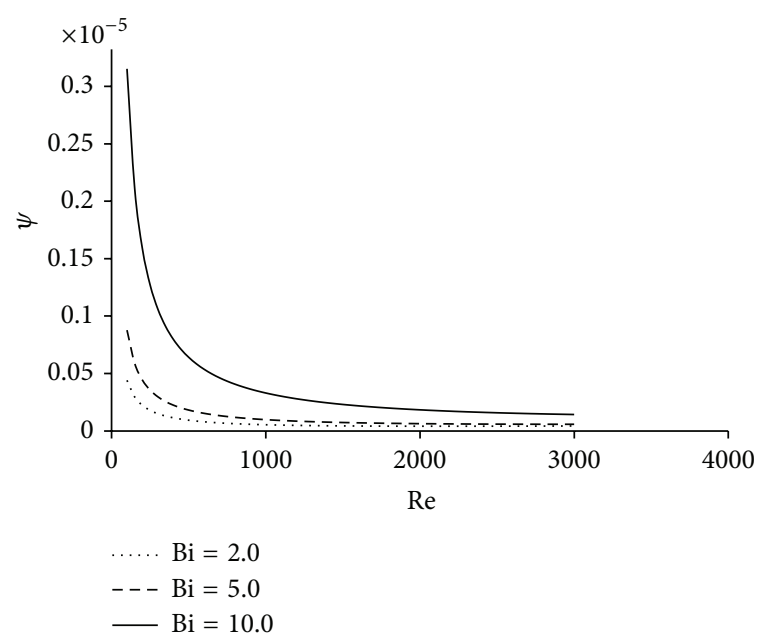

(a)

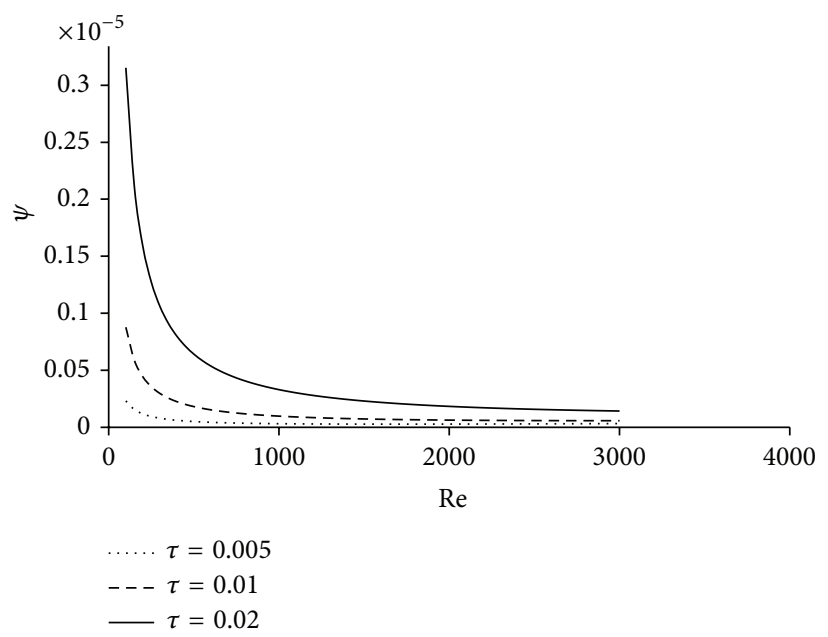

(b)

FIGURE 3: Variation of dimensionless entropy generation $\psi$ of ethylene glycol with Re for different values of (a) Bi (b) $\tau$.

in $\tau$ causes the heat transfer to the fluid to increase, which leads to an increase in temperature gradients and hence in $\psi$.

Figure 3 shows the variation of $\psi$ with Re for different values of (a) Bi and (b) $\tau$ for ethylene glycol. Similar trends, as observed for water, can be observed here.

3.2. Effect on Be. Figure 4 shows the variation of Be with Re for different values of (a) Bi and (b) $\tau$ for water. As can be seen from the figure, the value of Bejan number is very high (close to 1). This means that heat transfer irreversibility contributes almost $100 \%$ to the total entropy generation. This is corroborated by Ben-Mansour and Sahin [17] for circular pipes and by Jarungthammachote [6] for hexagonal pipes. We can also see from the figures that Be decreases with increase in Re. This is because, as pointed out in the previous section, the temperature gradients inside the fluid decrease with increase in Re. This leads to a decrease in heat transfer irreversibility. Moreover, the irreversibility due to pressure difference 


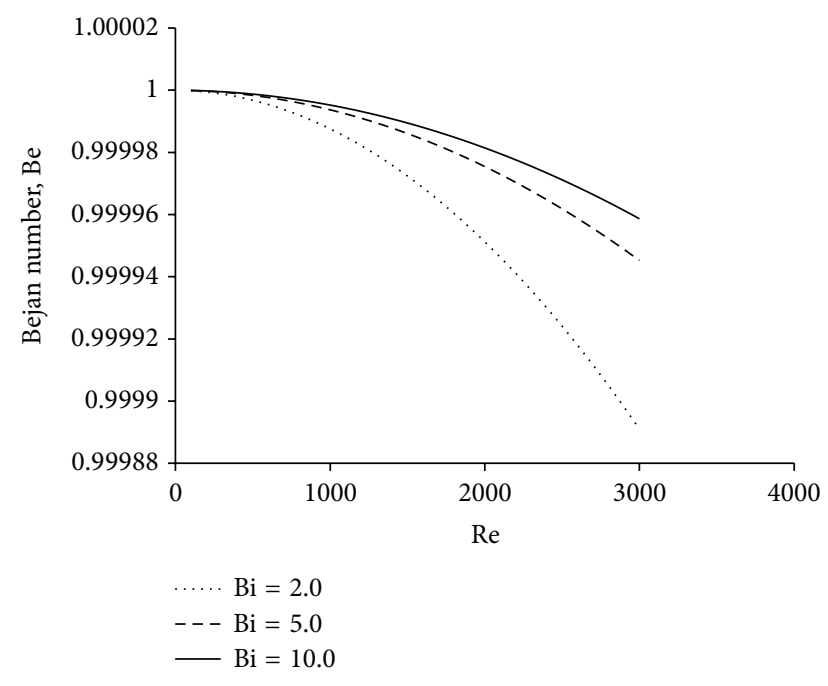

(a)

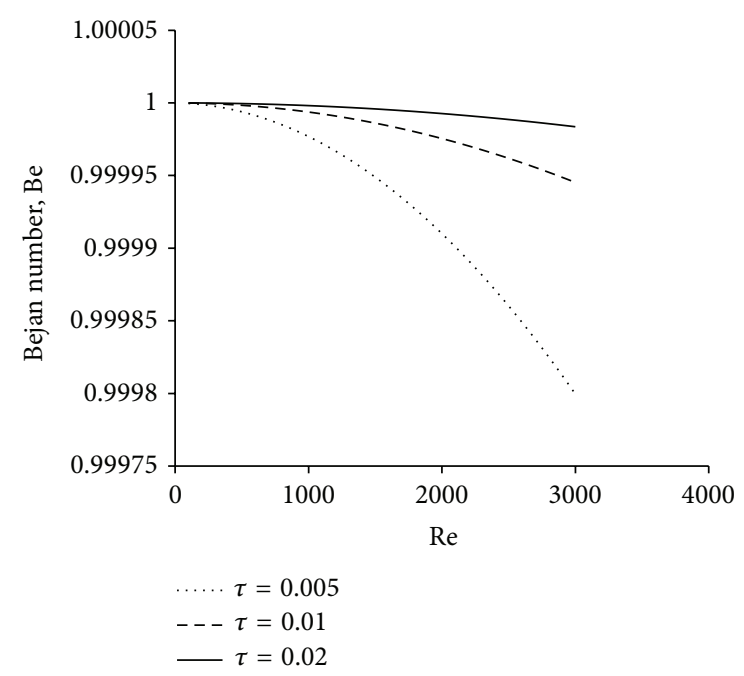

(b)

FIGURE 4: Variation of Bejan number of water with Re for different values of (a) Bi and (b) $\tau$.

increases with increase in Re. Due to these two reasons, Be decreases with increase in Re.

We can also see from Figure 4(a) that, as Bi increases, Be increases for a fixed Re. From Figure 4(b), we see that, as $\tau$ increases, $\mathrm{Be}$ increases. The result of these two figures can be explained on the basis of heat transfer irreversibility. As Bi or $\tau$ increases, the heat transfer to the fluid increases, due to which temperature gradients inside the fluid increase. This leads to increase in heat transfer irreversibility, which further leads to increase in Be.

Figure 5 shows the variation of Be with Re for different values of (a) Bi and (b) $\tau$ for ethylene glycol. Trends, similar to the case of water, can also be observed for ethylene glycol.

3.3. Effect on PPR. Figure 6 shows the variation of PPR with Re for different values of (a) Bi and (b) $\tau$ for water. As can be seen from the figure, for very small values of Re, PPR tends

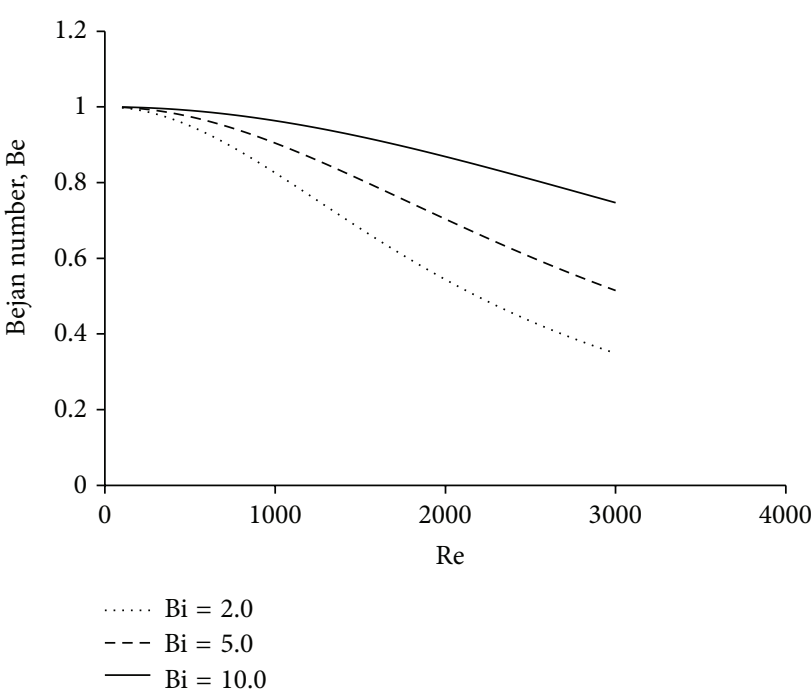

(a)

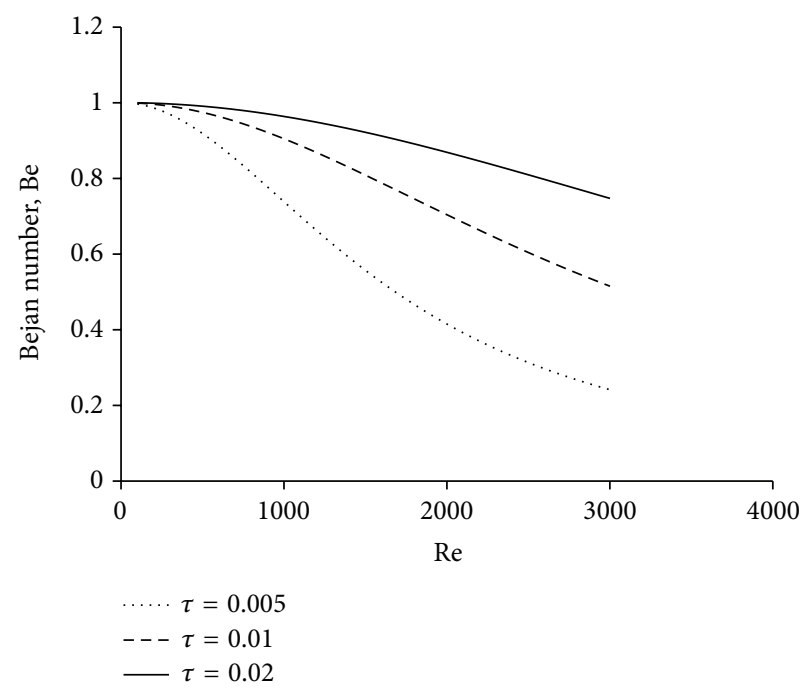

(b)

FIGURE 5: Variation of Bejan number of ethylene glycol with Re for different values of (a) Bi and (b) $\tau$.

to 0 . This is because, as $\operatorname{Re} \rightarrow 0$, there is no flow and so no pumping power is required. As Re increases, PPR increases, because, to maintain a flow at higher Re, more pumping power is required. Figure 6(a) shows that, as Bi increases, the PPR decreases. This is because, as $\mathrm{Bi}$ increases, $\widehat{\mathrm{N}} \mathrm{u}$ increases (see Table 1), which means more heat transfer to the fluid for the same Re. This leads to decrease in PPR. For the same reason, PPR decreases with increase in $\tau$, as shown in Figure 6(b).

Figure 7 shows the variation of PPR with Re for different values of (a) Bi and (b) $\tau$ for ethylene glycol. Trends, similar to the case of water, can be observed.

3.4. Comparison between Two Fluids. The comparison of total entropy generation, entropy generation due to heat transfer, and entropy generation due to pressure between water and 


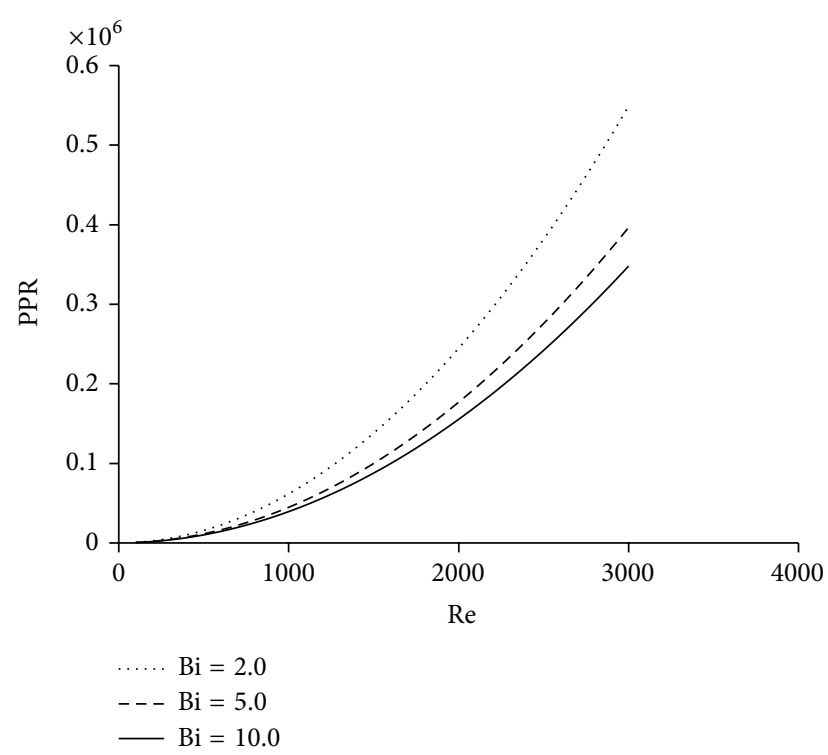

(a)

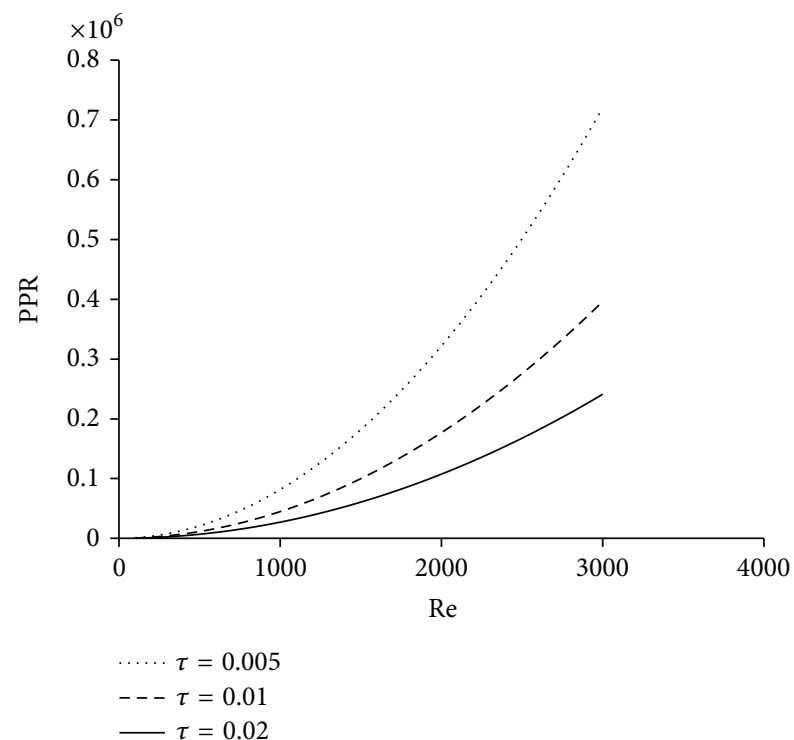

(b)

FIGURE 6: Variation of PPR of water with Re for different values of (a) Bi and (b) $\tau$.

ethylene glycol for the same mass flow rate, same Biot number, and same dimensionless temperature difference is shown in Table 3.

As can be seen from Table 3 , the total entropy generated and entropy generated due to heat transfer are higher for water while the entropy generated due to pressure is higher for ethylene glycol.

To investigate the reason for this trend, (9) must be paid attention to. For the same mass flow rate, the temperature at the outlet depends on $k / C_{p}$. Even though $C_{p}$ of water is higher than that of ethylene glycol, the $k$ for water is still higher, with the result that $k / C_{p}$ is higher for water. So $T_{\text {out }}$ for water is higher than $T_{\text {out }}$ for ethylene glycol. Due to this,

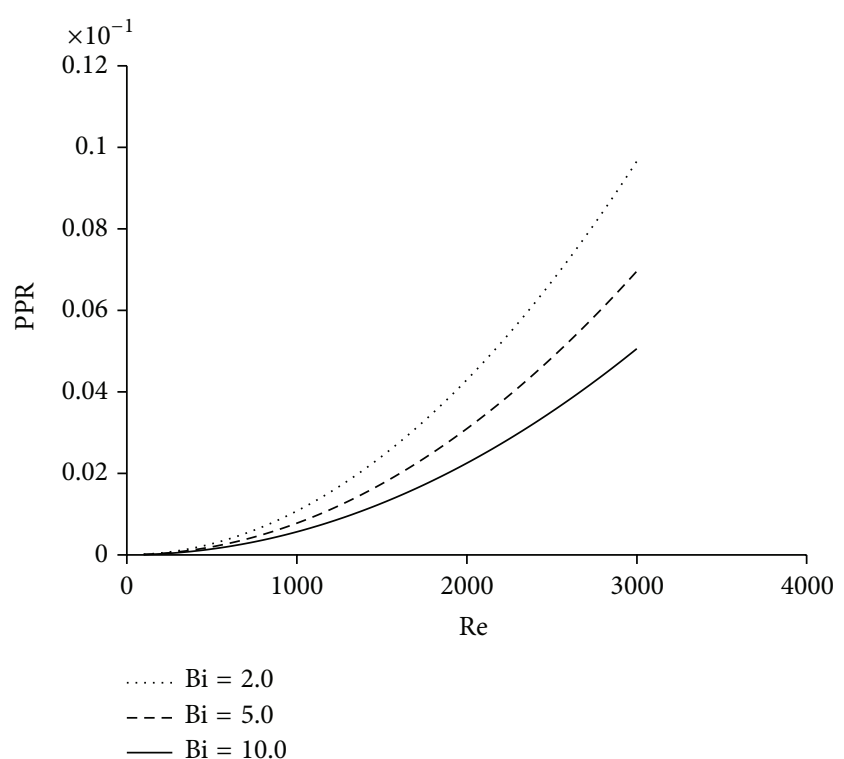

(a)

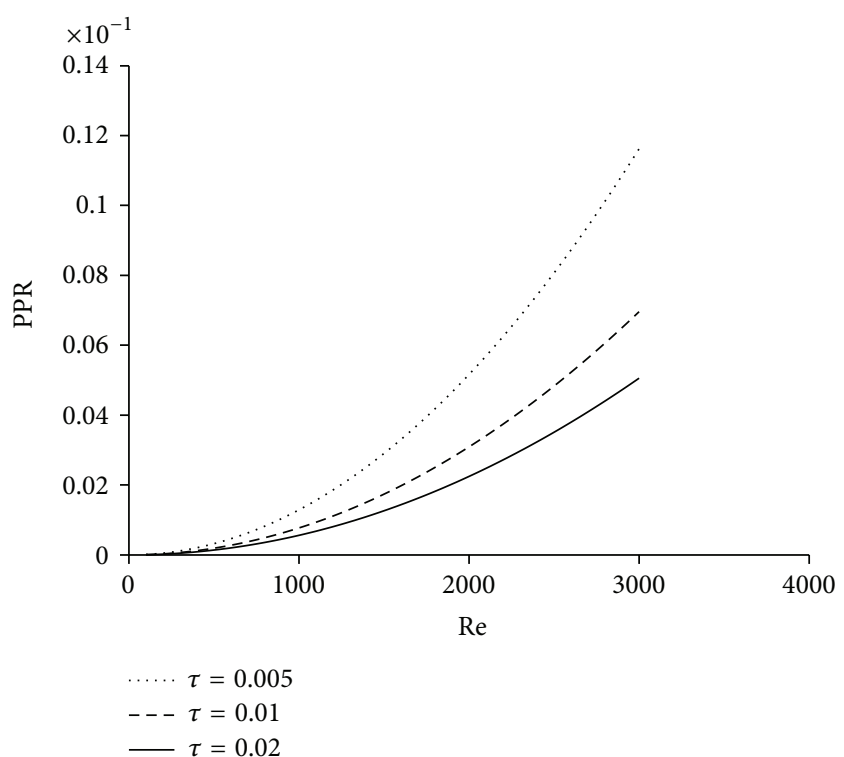

(b)

FIgURE 7: Variation of PPR of ethylene glycol with Re for different values of (a) Bi and (b) $\tau$.

temperature gradients inside the water are higher which leads to higher entropy generation for water than for ethylene glycol.

Entropy generation due to pressure is higher for ethylene glycol than that of water. This is because the viscosity of ethylene glycol is much higher than that of water.

3.5. Comparison with Uniform Wall Temperature. Figure 8 shows the comparison of (a) $\psi$ and (b) PPR between the circular duct with uniform wall temperature and circular duct immersed in an isothermal fluid. For comparison purpose, the temperature of isothermal external fluid is kept same as 
TABLE 3: Comparison of entropy generation due to temperature difference and entropy generation due to pressure for water and ethylene glycol.

\begin{tabular}{lcccccc}
\hline $\begin{array}{l}\dot{m} \\
(\mathrm{~kg} / \mathrm{s})\end{array}$ & $\begin{array}{c}\dot{S}_{\text {gen,Total }} \times 10^{3} \\
(\mathrm{~W} / \mathrm{K})\end{array}$ & $\begin{array}{c}\text { Water } \\
\dot{S}_{\text {gen, } \Delta T} \times 10^{3}\end{array}$ & $\begin{array}{c}\dot{S}_{\text {gen, } \Delta P} \times 10^{6} \\
(\mathrm{~W} / \mathrm{K})\end{array}$ & $\begin{array}{c}\dot{S}_{\text {gen,Total }} \times 10^{3} \\
(\mathrm{~W} / \mathrm{K})\end{array}$ & $\begin{array}{c}\text { Ethylene glycol } \\
\dot{S}_{\text {gen, } \Delta T} \times 10^{3} \\
(\mathrm{~W} / \mathrm{K})\end{array}$ & $\begin{array}{c}\dot{S}_{\text {gen, } \Delta P} \times 10^{6} \\
(\mathrm{~W} / \mathrm{K})\end{array}$ \\
\hline 0.05 & 0.839260096 & 0.839254054 & 0.006042 & 0.159089483 & 0.159012754 \\
0.06 & 0.847344381 & 0.847335678 & 0.008703 & 0.159603359 & 0.159492849 & 0.076729 \\
0.07 & 0.853184312 & 0.853172464 & 0.011848 & 0.159987558 & 0.159837123 & 0.196506 \\
0.08 & 0.857601029 & 0.857585551 & 0.015478 & 0.160292650 & 0.160096145 & 0.248721 \\
0.09 & 0.861058756 & 0.861039165 & 0.019591 & 0.160546880 & 0.160298159 & 0.307081 \\
\hline
\end{tabular}

TABLE 4: Comparison of dimensionless entropy generation $\psi$ with PPR for water and ethylene glycol.

\begin{tabular}{lcccc}
\hline \multirow{2}{*}{ Re } & \multicolumn{2}{c}{ Water } & \multicolumn{2}{c}{ Ethylene glycol } \\
& $\psi$ & PPR & $\psi$ & PPR \\
\hline 100 & $2.62 E-05$ & $5.63 E-10$ & $8.78 E-07$ & $7.76 E-06$ \\
500 & $8.08 E-06$ & $1.15 E-08$ & $1.82 E-07$ & 0.000193 \\
1000 & $4.28 E-06$ & $4.48 E-08$ & $9.8 E-08$ & 0.000774 \\
1500 & $2.91 E-06$ & $9.99 E-08$ & $7.33 E-08$ & 0.00174 \\
2000 & $2.21 E-06$ & $1.77 E-07$ & $6.33 E-08$ & 0.003094 \\
2500 & $1.78 E-06$ & $2.76 E-07$ & $5.91 E-08$ & 0.004834 \\
3000 & $1.49 E-06$ & $3.96 E-07$ & $5.8 E-08$ & 0.00696 \\
\hline
\end{tabular}

uniform wall temperature. As can be seen from the figure, $\psi$ is higher for uniform wall temperature condition than for isothermal external fluid condition, while PPR is lower for uniform wall temperature condition than for isothermal external fluid condition; other parameters are kept the same.

While comparing $\psi$ and PPR for different types of boundary conditions, the question arises: which type of boundary conditions is more suitable? To answer this, it should be noted that the total exergy loss in the pipe is the sum of $\psi$ and PPR. As can be seen from Table 4, for less viscous fluids like water, $\psi$ is much higher than PPR, a trend which becomes more pronounced for smaller Re. But more viscous fluids like ethylene glycol, on the other hand, have higher PPR than $\psi$. From this, it can be concluded that, for fluids whose viscosity is low, isothermal external fluid boundary condition should be preferred, while, for more viscous fluids, like ethylene glycol, uniform wall temperature boundary condition is to be preferred.

\section{Conclusions}

Dimensionless entropy generation, Be, and PPR of laminar flow in a circular tube immersed in an isothermal fluid are studied in this paper. The conclusions obtained can be summarised as follows.

(1) With increase in Re, both the dimensionless entropy generation and Be decrease, while PPR increases.

(2) With increase in $\mathrm{Bi}$, the dimensionless entropy generation and Be increase, while PPR decreases.
(3) With increase in $\tau$, the dimensionless entropy generation and Be increase, while PPR decreases.

(4) The entropy generated due to heat transfer is more for water than for ethylene glycol, while the entropy generated due to pressure is more for ethylene glycol than for water, for the same mass flow rate.

(5) PPR for ethylene glycol is higher than that for water, since ethylene glycol is more viscous than water.

(6) For more viscous fluids like ethylene glycol, uniform wall temperature boundary condition should be preferred, since it leads to less PPR. For less viscous fluid like water, a tube immersed in an isothermal fluid boundary condition should be preferred, since it leads to less entropy generation.

The values of $\widehat{\mathrm{Nu}}$ and $f$ used to derive the expression for dimensionless entropy generation have been taken from [12] and [16], respectively. In [12], the value of $\widehat{\mathrm{N}} \mathrm{u}$ has been derived numerically, while in [16] the value of $f$ has been derived analytically and verified experimentally. Thus, the margin of probable error in the values of $\widehat{\mathrm{Nu}}$ and $f$ is very less. In the highly unlikely case of small errors creeping in the values of $\widehat{\mathrm{Nu}}$ and $f$, the trends of dimensionless entropy generation presented here will remain unchanged qualitatively.

\section{Nomenclature}

A: Cross-sectional area $\left(\mathrm{m}^{2}\right)$

Be: Bejan number

Bi: Biot number $h_{e} r / k$

$C_{p}:$ Specific heat capacity $(\mathrm{J} / \mathrm{kg} \mathrm{K})$

$\mathrm{CV}$ : Control volume

$D$ : Cross-sectional diameter $(\mathrm{m})$

Ec: Eckert number (defined in (21))

$f:$ Friction factor

FF: Dimensionless entropy generated due to fluid friction

$h$ : Internal heat transfer coefficient $\dot{q}^{\prime \prime} /\left(T_{\text {wall }}-T\right)$ $\left(\mathrm{W} / \mathrm{m}^{2} \mathrm{~K}\right)$

$\widehat{h}$ : Overall heat transfer coefficient $\dot{q}^{\prime \prime} /\left(T_{e}-T\right)$ $\left(\mathrm{W} / \mathrm{m}^{2} \mathrm{~K}\right)$

$h_{e}$ : External heat transfer coefficient $\dot{q}^{\prime \prime} /\left(T_{e}-T_{\text {wall }}\right)$ $\left(\mathrm{W} / \mathrm{m}^{2} \mathrm{~K}\right)$

$k$ : Thermal conductivity (W/m K) 


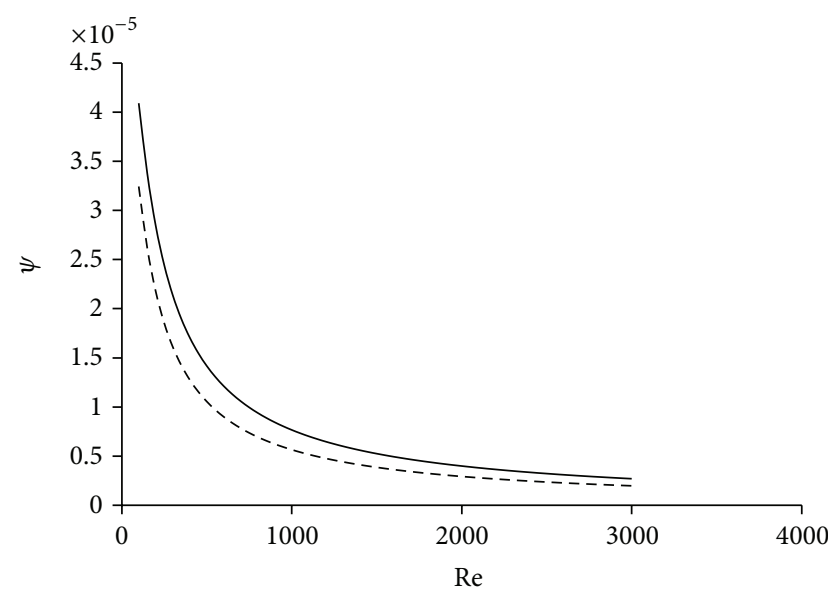

- Uniform wall temperature - - - Isothermal external fluid

(a)

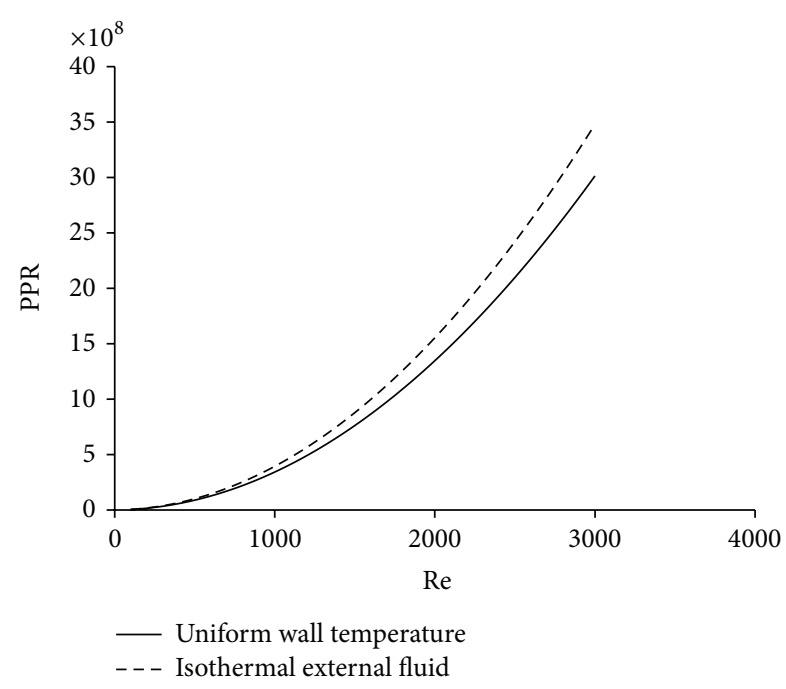

(b)

FIGURE 8: Comparison of (a) $\psi$ and (b) PPR for different boundary conditions.

\footnotetext{
$L: \quad$ Duct length (m)

$\dot{m}$ : Mass flow rate $(\mathrm{kg} / \mathrm{s})$

$\mathrm{Nu}$ Internal Nusselt number $h D / k$

$\widehat{N} u:$ Overall Nusselt number $\widehat{h} D / k$

$P: \quad$ Pressure $(\mathrm{Pa})$

PPR: Pumping power to heat transfer ratio

(defined in (31))

Pr: Prandtl number

$\dot{q}^{\prime \prime}$ : Local heat flux $\left(\mathrm{W} / \mathrm{m}^{2}\right)$

$\dot{q}^{\prime}: \quad$ Heat rate per unit length $(\mathrm{W} / \mathrm{m})$

$\dot{Q}: \quad$ Heat rate $(\mathrm{W})$

$r$ : Cross-section radius $(\mathrm{m})$

Re: Reynolds number

$s: \quad$ Specific entropy $(\mathrm{J} / \mathrm{kg} \cdot \mathrm{K})$

$\dot{S}: \quad$ Entropy rate $(\mathrm{W} / \mathrm{K})$

St: $\quad$ Stanton number (defined in (5))
}

T: Temperature $(\mathrm{K})$

$U$ : Average fluid velocity $(\mathrm{m} / \mathrm{s})$.

\section{Greek Letters}

$\alpha: \quad$ Dimensionless parameter (defined in (19))

$\tau$ : Dimensionless temperature difference (defined in (20))

$\lambda$ : Dimensionless length (defined in (6))

$\lambda_{L}$ : Dimensionless length (defined in (8))

$\psi$ : Dimensionless entropy generation (defined in (22))

$\Delta P$ : Pressure drop $(\mathrm{Pa})$

$\rho: \quad$ Fluid density $\left(\mathrm{kg} / \mathrm{m}^{3}\right)$

$\mu: \quad$ Fluid viscosity (Ns/m²)

$\pi_{1}$ : Dimensionless parameter (defined in (26))

$\pi_{2}$ : Dimensionless parameter (defined in (27)).

\section{Subscripts}

avg: Average

e: $\quad$ External

gen: Generated

$i$ : $\quad$ Inlet

out: Outlet

ref: Reference

Total: Total

$w$ : Water

wall: Wall

$\Delta P: \quad$ Pressure difference

$\Delta T: \quad$ Temperature difference.

\section{Acknowledgment}

Sincere thanks are due to Dr. T. K. Vashist of Infotech Enterprises Limited, Bangalore, for his contribution and guidance towards revising the paper.

\section{References}

[1] A. Bejan, "Second law analysis in heat transfer," Energy, vol. 5, no. 8-9, pp. 721-732, 1980.

[2] A. Bejan, Entropy Generation through Heat and Fluid Flow, John Wiley \& Sons, New York, NY, USA, 1982.

[3] A. Z. Sahin, "Second law analysis of laminar viscous flow through a duct subjected to constant wall temperature," Journal of Heat Transfer, vol. 120, pp. 76-83, 1998.

[4] A. Z. Şahin, "Irreversibilities in various duct geometries with constant wall heat flux and laminar flow," Energy, vol. 23, no. 6, pp. 465-473, 1998.

[5] A. Z. Şahin, "A second law comparison for optimum shape of duct subjected to constant wall temperature and laminar flow," Heat and Mass Transfer/Waerme- und Stoffuebertragung, vol. 33, no. 5-6, pp. 425-430, 1998.

[6] S. Jarungthammachote, "Entropy generation analysis for fully developed laminar convection in hexagonal duct subjected to constant heat flux," Energy, vol. 35, no. 12, pp. 5374-5379, 2010.

[7] T. H. Ko and K. Ting, "Entropy generation and thermodynamic optimization of fully developed laminar convection in a helical 
coil," International Communications in Heat and Mass Transfer, vol. 32, no. 1-2, pp. 214-223, 2005.

[8] I. Dağtekin, H. F. Öztop, and A. Z. Şahin, "An analysis of entropy generation through a circular duct with different shaped longitudinal fins for laminar flow," International Journal of Heat and Mass Transfer, vol. 48, no. 1, pp. 171-181, 2005.

[9] S. Mahmud and R. A. Fraser, "Inherent irreversibility of channel and pipe flows for non-Newtonian fluids," International Communications in Heat and Mass Transfer, vol. 29, no. 5, pp. 577$587,2002$.

[10] S. Mahmud and R. A. Fraser, "Second law analysis of forced convection in a circular duct for non-Newtonian fluids," Energy, vol. 31, no. 12, pp. 2226-2244, 2006.

[11] Y.-M. Hung, "Viscous dissipation effect on entropy generation for non-Newtonian fluids in microchannels," International Communications in Heat and Mass Transfer, vol. 35, no. 9, pp. 1125-1129, 2008.

[12] E. M. Sparrow and S. V. Patankar, "Relationships amoung boundary conditions and nusselt numbers for thermally developed duct flows," Journal of Heat Transfer, vol. 99, no. 3, pp. 483485, 1977.

[13] S. Paoletti, F. Rispoli, and E. Sciubba, "Calculation of exergetic losses in compact heat exchanger passages," Tech. Rep. ASME AES- 10, 1989.

[14] F. S. Sherman, Viscous Flow, McGraw Hill, New York, NY, USA, 1990.

[15] F. P. Incropera and D. P. DeWitt, Fundamentals of Heat and Mass Transfer, John Wiley \& Sons, Singapore, 5th edition, 2006.

[16] R. W. Fox and A. T. McDonald, Introduction to Fluid Mechanics, John Wiley \& Sons, Singapore, 5th edition, 2004.

[17] R. Ben-Mansour and A. Z. Sahin, "Entropy generation in developing laminar fluid flow through a circular pipe with variable properties," Heat and Mass Transfer/Waerme- und Stoffuebertragung, vol. 42, no. 1, pp. 1-11, 2005. 

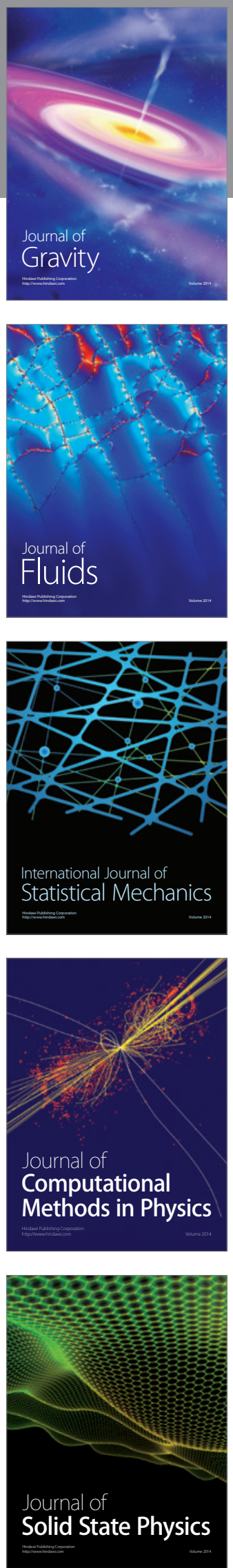

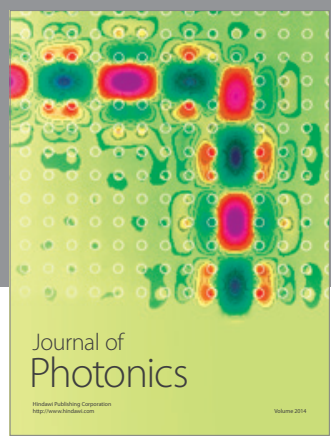

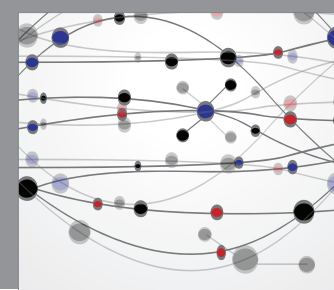

The Scientific World Journal

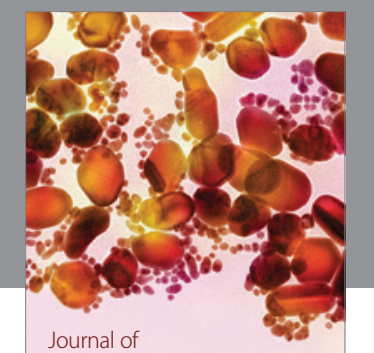

Soft Matter
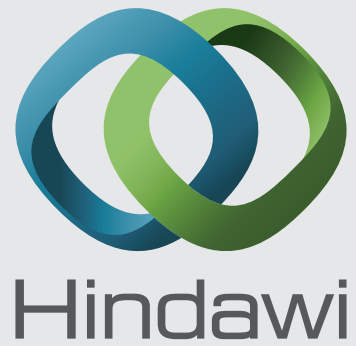

Submit your manuscripts at

http://www.hindawi.com
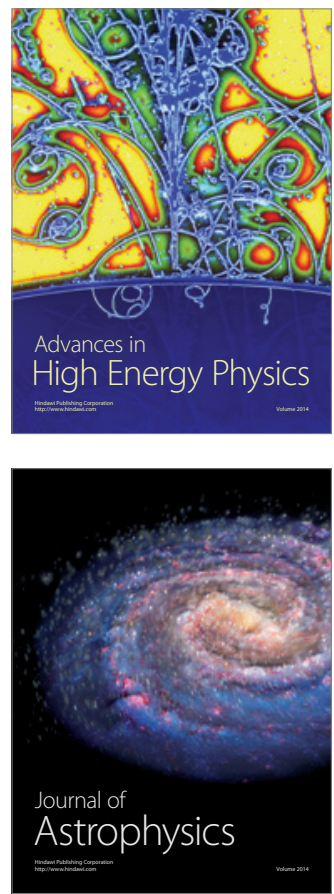
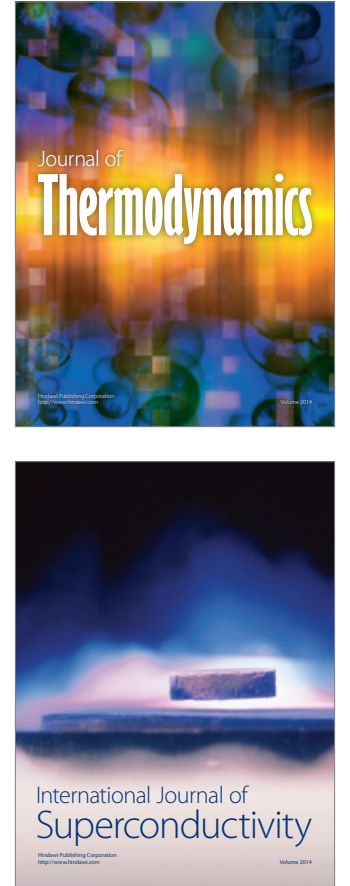
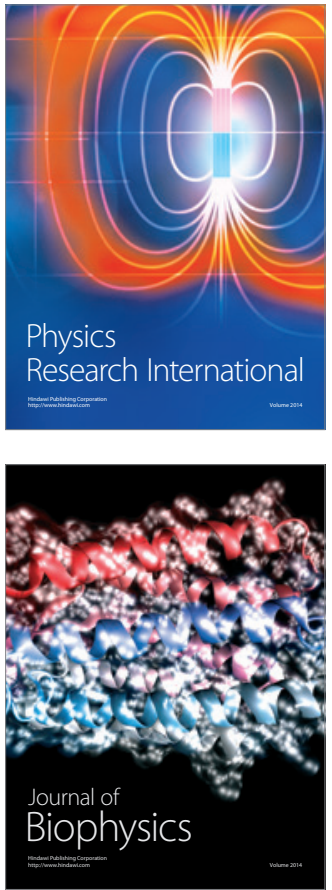
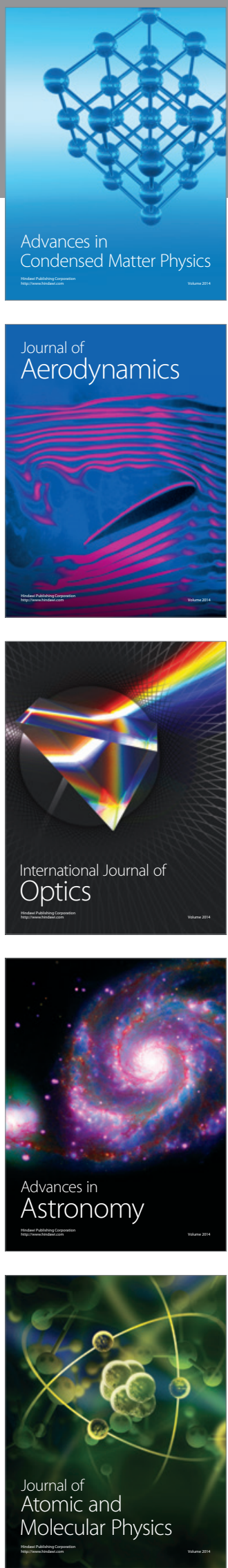